\title{
O ESPORTE ADAPTADO NA ESCOLA: REFLEXÕES A PARTIR DA PRODUÇÃO ACADÊMICA NACIONAL
}

\section{THE SPORT ADAPTED AT SCHOOL: REFLECTIONS FROM THE NATIONAL ACADEMIC PRODUCTION}

ANTUNES, Marcelo Moreira1

\begin{abstract}
RESUMO
O esporte adaptado tem se desenvolvido largamente em diferentes setores da sociedade. Desde políticas públicas de saúde e lazer, até sua inserção na Educação Básica. O interesse por essa temática tem crescido nos últimos anos e a produção científica tem demonstrado essa expansão. A partir desse contexto, o presente estudo objetivou fazer uma análise da produção acadêmica brasileira que trata do esporte adaptado na escola, especificamente as dissertações e teses produzidas pelos programas de pós-graduação em Educação Física. Delimitou-se o período de 2007 a 2017 com recorte temporal da pesquisa. A busca foi realizada no Catalogo de Dissertações e Teses da CAPES. Foram encontradas sete dissertações e três teses que atenderam os critérios de inclusão e exclusão. Como resultados, foram identificadas três categorias de análise dos estudos que demonstraram maior concentração em pesquisas diagnósticas. Pode-se concluir que há grande lacuna de estudos que tratam de propostas para intervenção pedagógica com os esportes adaptados na escola para alunos com ou sem deficiência.
\end{abstract}

PALAVRAS-ChAVe: Esporte adaptado; Educação inclusiva; Educação física adaptada.

\begin{abstract}
Adapted sport has developed widely in different sectors of society: from public health and leisure policies to its insertion in basic education. Interest in this subject has grown in recent years and scientific production has reflected this increase. From this context this study attempted to analyze Brazilian academic production that refers to adapted sport in school, specifically dissertations and theses produced by physical education post-graduate programs. The research established the years 2007 to 2017 as its time period. The search was undertaken in the Catalog of Theses and Dissertations of CAPES. Seven dissertations and three theses were found that met inclusion and exclusion criteria. As a result, one could identify three categories of study analysis that showed greater concentration in diagnostic research. One can conclude that there is a large gap in studies that deal with adapted sports pedagogical intervention proposals for students with or without disabilities.
\end{abstract}

KeYwORDS: Adapted sport; Inclusive education; Adapted physical education.

1 Professor Adjunto da Universidade Federal Fluminense (UFF). Niterói, RJ, Brasil. ORCID: http://orcid.org/0000-0003-0444-1332 e-mail: antunesmm@gmail.com 
DOI: $10.12957 /$ e-mosaicos.2020.42690

\section{INTRODUÇÃO}

O esporte para pessoas com deficiência apresenta na atualidade significativo crescimento em diferentes setores da sociedade global, como no esporte de alto rendimento, no lazer, nas políticas de inclusão e saúde pública e na educação (PATATAS; BOSSCHER; LEGG, 2018). No Brasil, esses esportes, sejam eles paralímpicos ou não, têm se difundido expressivamente na última década. Desde os jogos Paralímpicos no Rio de Janeiro em 2007, percebeu-se o grande potencial dessas modalidades para um público ainda pouco atendido. Os resultados expressivos de brasileiros em competições internacionais têm revelado esse grande potencial. E, como resultado dessa percepção, a quantidade de estudos sobre os esportes para pessoas com deficiência tem se desenvolvido de maneira expressiva.

Parte desse resultado, além do interesse crescente pelo tema, indica um claro aumento de programas de pós-graduação que abrem linhas de pesquisa para tratar de atividades motoras para pessoas com deficiência e seus aspectos educacionais e de inclusão. $E$, nesse universo diverso de modalidades esportivas, estão as que podem compor o currículo da educação física escolar.

A partir desse novo cenário que destaca as artes marciais e dos esportes de combate como práticas possíveis para pessoas com deficiência, e da condição do esporte moderno como significativo fenômeno social da atualidade, o presente estudo se insere como uma possibilidade de avaliar como essas práticas estão sendo estudadas com base nos programas de pós-graduação em Educação Física no Brasil.

\section{MÉTODO}

O presente estudo se constitui como uma revisão da literatura a partir do entendimento de Thomas, Nelson e Silverman (2012) que afirmam que esse tipo de pesquisa procura realizar um mapeamento do estado da arte de um determinado assunto. Como fonte de dados, foram usadas teses e dissertações produzidas em programas de Pós-graduação acadêmicos que tratam dos esportes para pessoas com deficiência em ambiente escolar. Esses documentos estão disponíveis no Catálogo de Teses e Dissertações da Coordenação de Aperfeiçoamento de Pessoal de Nível Superior (CAPES) no endereço eletrônico http://catalogodeteses.capes.gov.br/catalogo-teses/\#!/. Esse catálogo agrupa as teses e dissertações de programas de pós-graduação reconhecidos e registra os trabalhos produzidos desde 1987. O recorte temporal delineado foi o período de 10 anos, definido entre 2007 e 2017. 0 ano de 2007 foi selecionado, pois se configurou como marco para o Brasil no esporte adaptado pelo sucesso em sua participação nos Jogos Paralímpicos e também por sediar a edição dos jogos nesse ano. 
DOI: $10.12957 /$ e-mosaicos.2020.42690

Para a busca pelas teses e dissertações, foram utilizadas as seguintes palavraschave: Esporte adaptado; Atividade motora adaptada; Educação física adaptada; Esporte paralímpico; Esporte paraolímpico.

Como critérios de inclusão foram considerados as teses e dissertações produzidas por programas de pós-graduação da grande área da saúde, da área Educação Física e que tratassem de esportes para pessoas com deficiência em ambiente escolar, paralímpicos ou não, de forma curricular ou extracurricular. Além disso, os estudos devem se concentrar em aspectos pedagógicos, curriculares e inclusivos. Como critério de exclusão considerou-se estudos realizados em conjunto com esportes não adaptados ou destinados a pessoas sem deficiência.

\section{RESULTADOS E DISCUSSÃo}

Apesar de os debates sobre a inclusão da pessoa com deficiência ter se intensificado a partir de 1994 com a promulgação da Declaração de Salamanca, da qual o Brasil é um dos signatários, as atividades esportivas para pessoas com deficiência na escola ainda são muito incipientes. Mesmo com um significativo número de estudos na área do esporte adaptado e da atividade motora adaptada, a maioria deles não versa sobre a escola e sua oferta de atividades para esse público. Percebese esse fato nos resultados preliminares da busca no Catálogo de Teses e Dissertações da Capes. Foram encontradas 14763 ocorrências de dissertações de mestrado e teses de doutorado, divididas pelas palavras de busca como apresentado na tabela abaixo.

Tabela 1: Ocorrências de resultados por palavra-chave

\begin{tabular}{|c|c|c|c|}
\hline Palavra-Chave & Ocorrência & Nível & $(\%)$ \\
\hline \multirow{2}{*}{ Esporte adaptado } & \multirow{2}{*}{2345} & $1843(\mathrm{M})$ & \multirow{2}{*}{14,38} \\
\hline & & $502(\mathrm{D})$ & \\
\hline \multirow{2}{*}{ Atividade motora adaptada } & \multirow{2}{*}{3299} & $2684(M)$ & \multirow{2}{*}{20,24} \\
\hline & & $615(\mathrm{D})$ & \\
\hline \multirow{2}{*}{ Educação física adaptada } & \multirow{2}{*}{6088} & $4964(\mathrm{M})$ & \multirow{2}{*}{37,35} \\
\hline & & $1124(\mathrm{D})$ & \\
\hline \multirow{2}{*}{ Esporte paralímpico } & \multirow{2}{*}{2286} & $1800(\mathrm{M})$ & \multirow{2}{*}{14,02} \\
\hline & & $486(D)$ & \\
\hline \multirow{2}{*}{ Esporte paraolímpico } & \multirow{2}{*}{2282} & $1796(\mathrm{M})$ & \multirow{2}{*}{14,00} \\
\hline & & $486(\mathrm{D})$ & \\
\hline \multirow{2}{*}{ TOTAIS } & \multirow{2}{*}{16300} & $13087(\mathrm{M})$ & \multirow{2}{*}{100} \\
\hline & & 3213 (D) & \\
\hline
\end{tabular}

Legenda: (M) Mestrado; (D) Doutorado. Fonte: Dados da pesquisa

O grande número de estudos não reflete seu real status, pois, muitos deles se sobrepõem em função da ocorrência duplicada, ou mais, dos termos de busca em um mesmo estudo. Assim, a multiplicidade de estudos que reaparecem em buscas específicas ocorre com grande frequência. Outra situação importante referente ao grande número de estudos encontrados nessa primeira busca é o fato de que as palavras-chave podem ser relacionadas a qualquer parte do texto e não necessária 
DOI: $10.12957 /$ e-mosaicos.2020.42690

mente circunscreve o tema fim da busca. Portanto, estudos com temas como ergonomia, psicologia, fisiologia, reabilitação, sociologia, gestão, treinamento, entre outros, podem conter as palavras selecionadas para a busca, não representando o tema foco desse estudo.

Após a aplicação dos critérios de inclusão e exclusão descritos anteriormente para o presente estudo, foram analisados 10 estudos, sendo 7 de mestrado e 3 de doutorado. A distribuição dos estudos ao longo da linha do tempo e seu nível de pósgraduação estão representados na tabela a seguir.

Tabela 2: Ocorrência dos estudos selecionados

\begin{tabular}{|c|c|c|c|}
\hline ANO & 2013 & 2016 & 2017 \\
\hline Nível & Mestrado (4) & Doutorado (1) & $\begin{array}{c}\text { Mestrado (3) } \\
\text { Doutorado (2) }\end{array}$ \\
\hline
\end{tabular}

Fonte: Dados da pesquisa

Mesmo que o recorte temporal inclua estudos apresentados a partir do ano de 2007 e que diversos estudos tenham sido realizados nesse período, apenas em 2013 se localizam os primeiros com esporte adaptado em ambiente escolar. Esses estudos se distribuíram predominantemente pelas regiões Sul e Sudeste, como é demonstrado no quadro a seguir.

\begin{tabular}{|c|c|c|c|}
\hline Região & Instituição & Ocorrência & Nível \\
\hline \multirow{2}{*}{ Sul } & Universidade Estadual de Maringá & 1 & Doutorado \\
\hline & Universidade Federal do Paraná & 2 & Mestrado \\
\hline \multirow{5}{*}{ Sudeste } & Universidade de São Paulo & 2 & Doutorado \\
\hline & Universidade Estadual de Campinas & 2 & Mestrado \\
\hline & Universidade Federal de Juiz de Fora & 1 & Mestrado \\
\hline & Universidade Federal do Espírito Santo & 1 & Mestrado \\
\hline & Universidade Salgado de Oliveira & 1 & Mestrado \\
\hline
\end{tabular}

Fonte: Dados da pesquisa

A partir do que é apresentado no Quadro 1, percebe-se que os estudos sobre o esporte adaptado se concentram na região Sudeste, com sete ocorrências, e com três estudos apenas ocorrendo na região Sul. Isoladamente, as instituições que mais produziram estudos sobre o tema foram a Universidade de São Paulo (USP) com duas teses, a Universidade Estadual de Campinas (UNICAMP) com duas dissertações de mestrado e a Universidade Federal do Paraná (UFPR) com a publicação de duas dissertações de mestrado. Em contrapartida, segundo dados atualizados da CAPES, existem 38 programas de pós-graduação em Educação Física no Brasil, distribuídos em 38 instituições de Ensino Superior, sendo 9 na região Sul, dezessete na região Sudeste, três na Centro-Oeste, seis na região Nordeste e nenhuma no Norte. Esse cenário geográfico demonstra uma concentração de estudos em regiões especificas que pode 
DOI: $10.12957 /$ e-mosaicos. 2020.42690

ser explicado pela proximidade de centros de desenvolvimento do esporte paralímpico e do esporte adaptado como o Centro de treinamento do Comitê Paralímpico Brasileiro em São Paulo e núcleos de desenvolvimento de estudo em atividade motora adaptada como o que se estabelece na UNICAMP. Portanto, ainda há um vasto campo para o desenvolvimento da temática em diversas instituições de pesquisa e em programas de pós-graduação no Brasil.

Os trabalhos analisados apresentaram distintas categorias e subcategorias de análise a partir dos pressupostos da Análise de Conteúdo de Bardin (2009). Foram identificadas as categorias estudos propositivo, diagnóstico e misto (diagnóstico e propositivo). $O$ estudo propositivo se refere às pesquisas que desenvolveram propostas de planejamento e intervenção utilizando o esporte adaptado para pessoas com deficiência. Os estudos diagnósticos são considerados aqueles que buscaram verificar e constatar uma determinada situação ou característica de um grupo de alunos, professores, práticas educativas ou outra condição relacionada ao esporte adaptado em ambiente escolar. As categorias e subcategorias são apresentadas no quadro a seguir. O misto engloba no mesmo estudo um diagnóstico do cenário e de práticas vigentes visando embasar uma proposição de intervenção.

Quadro 2 - Categorias e subcategorias dos estudos analisados

\begin{tabular}{|ccc|}
\hline Categorias & Subcategorias & Ocorrência \\
\hline Estudo Propositivo & Pesquisa de campo & 3 \\
Estudo Diagnóstico & Pesquisa de campo & 4 \\
\cline { 2 - 4 } & Análise documental & 2 \\
\hline Estudo Misto & Revisão da literatura e Pesquisa de \\
& campo & 1 \\
\hline
\end{tabular}

Fonte: Dados da pesquisa

Pode-se verificar que a maior concentração em estudos com o caráter diagnóstico buscou compreender as características dos alunos com deficiência, das próprias deficiências, a literatura que trata do tema e os documentos oficiais que orientam a prática educativa para esse público. Essa tendência pode indicar que ainda há uma compreensão superficial sobre a educação inclusiva, sobre o esporte adaptado na escola e também sobre as características dos próprios alunos com deficiência. Para Lopes e Fabris (2013), conhecer o ambiente escolar e suas particularidades é essencial para uma boa prática inclusiva. Desse modo, estudos diagnósticos auxiliam na compreensão das características do ambiente e dos atores do cenário educacional. 
DOI: $10.12957 / \mathrm{e}-\mathrm{mosaicos} .2020 .42690$

Esse conhecimento dos atores e cenários é fundamental para os processos de mudança necessários na escola. As mudanças se dão na escola a partir da modificação de todas as suas estruturas, incluindo o currículo, a avaliação, os conteúdos, os processos de tomada de decisões e as práticas pedagógicas. Uma reestruturação de todas as suas dimensões, incluindo as práticas extramuros que envolve toda a comunidade escolar, especificamente, o desconhecimento das características das diferentes deficiências impede uma estratégia pedagógica inclusiva (GORGATTI, 2005). A partir desse contexto, talvez se possa explicar a predominância de estudos diagnósticos e descritivos. Porém, mesmo nos estudos dessa categoria, há a predominância dos diagnósticos usando pesquisas de campo. Isso pode demonstrar que parte do conhecimento conceitual já está parcialmente absorvido, restando ainda a exploração dos atores em seus cenários de inserção.

Os estudos que se caracterizaram como sendo propositivo, apesar de serem em menor número, demonstram o início do processo de experimentação e transferência dos conhecimentos teóricos para a atuação prática. Esses estudos demonstram processos de reflexão sobre as características dos alunos e suas potencialidades, além de realizarem adaptações dos conteúdos, das formas de intervenção e nos processos avaliativos. Isso se estabelece como superação da barreira imposta aos alunos com deficiência como sinalizam diversos autores (GORGATTI, 2005; BRACHT; SOARES, 2005; SILVA; SANTIAGO; BERTONI, 2011). Para esses estudiosos, além disso, se configuram como obstáculos a uma educação física inclusiva o desconhecimento das características da deficiência, a não adaptação dos conteúdos, a má formação inicial ou continuada no que se refere a essa temática, a infraestrutura inadequada, a desarticulação dos diferentes setores da escola, além de a falta de acompanhamento de profissionais especializados.

\subsection{Categoria de estudos diagnósticos}

Nessa categoria, encontramos seis estudos que contemplaram três subcategorias: Pesquisa de campo, Revisão da literatura e Análise documental. Esses estudos são apresentados sinteticamente a seguir.

Santos (2017), em sua dissertação de mestrado, objetivou descrever e avaliar os fatores que podem influenciar o Transtorno do Desenvolvimento da Coordenação na primeira infância em creches e domicílios. Apesar de parte do estudo verificar esses fatores nos domićílios dos alunos, a maior coleta de dados foi realizada nas creches da rede pública de ensino. Inicialmente, o estudo avaliou 228 crianças entre 3 e 5 anos quanto a sua dimensão motora. Posteriormente, foi realizado um survey com responsáveis e professores de 42 crianças de 3 a 5 anos de ambos os sexos selecionados a partir da avaliação inicial. Os resultados demonstraram que os responsáveis percebem o problema motor nas crianças durante a realização de atividades acadêmicas, e os professores perceberam durante as atividades físicas. Essa diferença sugere o aumento de interlocução entre os responsáveis e a escola no 
DOI: $10.12957 /$ e-mosaicos.2020.42690

sentido de diminuir os efeitos negativos dessa condição em que as crianças se encontram.

Na dissertação de mestrado de Carvalho (2013), é apresentado um estudo qualitativo investigando as práticas pedagógicas que permitam a realização de uma educação inclusiva em atividades esportivas, recreativas e corporais. Para isso, a autora realizou quatro ações. A primeira realizou uma revisão da literatura sobre a inclusão de alunos com deficiência auditiva em ambiente escolar. A segunda ação avaliou como a língua de sinais permite maior interação entre os alunos e de que forma a prática da Língua Brasileira de Sinais (LIBRAS) possibilita maior facilidade em tarefas motoras. A terceira articulou as percepções de três alunos com deficiência e dois professores especialistas no que se refere à possibilidade de interação entre alunos com e sem deficiência auditiva durante as práticas de atividades físicas na escola. A quarta e última ação investigou sentidos e significados atribuídos por seis alunos participantes de um projeto de atividades corporais e artísticas que utiliza a LIBRAS em sua articulação com as atividades e alunos. Conclui que recursos materiais e visuais são importantes para educação de surdos, bem como atuação de profissionais qualificados. Entretanto, a cultura do aluno com deficiência auditiva deve ser valorizada. A LIBRAS se transforma em uma excelente ferramenta para o desenvolvimento do aluno, ouvinte ou não, e de sua inclusão em diferentes cenários das práticas corporais.

O estudo de análise documental de Silva (2017), que se constituiu em sua dissertação de mestrado, buscou compreender como algumas ações de entidades governamentais e de gestão do esporte nacional, a partir de seus documentos oficiais e legislação federal especifica - Lei de Incentivo ao Esporte ( $\left.n^{0} 11.438 / 2006\right)$, Lei Agnelo/Piva (no 10.264/2001) e o programa Bolsa Atleta (no 10.891/2004) impactaram no desenvolvimento do paradesporto educacional. Para isso, a autora analisou as ações do Ministério do Esporte e do Comitê Paralímpico Brasileiro, além dos dados do projeto Paralimpíadas Escolares. Como resultado dos dados analisados, foram encontradas duas conexões que se desdobram em efeitos distintos. A primeira é a relação do repasse de verbas para a realização das Paralimpíadas Escolares, e a segunda é a articulação entre o programa Bolsa Atleta e as Paralimpíadas Escolares. Na primeira, há uma falha na compreensão sobre as práticas e objetivos de um paradesporto escolar, principalmente quando se foca no alto rendimento como processo excludente. Na segunda conexão, há um benefício direto dos alunos que são contemplados pelo programa Bolsa atleta no que se refere à continuidade de sua carreira esportiva.

A dissertação de Cancella (2017) buscou, através de uma análise documental, verificar a oferta de esportes para alunos com deficiência no Instituto Federal do Paraná (IFPR), campus Paranaguá. Para isso, o autor realizou um levantamento bibliográfico no sentido de realizar uma análise histórica da legislação sobre o esporte no país e especificamente o esporte para pessoas com deficiência. Consultou e analisou as informações contidas no site da Rede Federal EPCT, no Plano de 
DOI: $10.12957 /$ e-mosaicos.2020.42690

Desenvolvimento Institucional (PDI) do IFPR (2014-2018), no Projeto Político Pedagógico (PPP) do campus Paranaguá e nos Planos de Ensino Curriculares da Educação Física (PECEFs). Estabeleceu ainda interlocução com a teoria de campo de Pierre Bourdieu na busca de identificar pontos de tensionamentos em relação à violência simbólica e à reprodução de modelos dominantes de ensino. Foram utilizadas para a análise dos dados três categorias: Oferta do Esporte; Cotas de Inclusão ou Exclusão; Reprodução ou Resistência. Os resultados indicam que o IFPR possui políticas diferenciadas que permitem a oferta de esportes na perspectiva inclusiva, representando um movimento de resistências aos modelos dominantes de ensino na Educação Básica no que se refere ao esporte escolar.

O estudo de doutorado de Goulardins (2016) investigou crianças em duas escolas do Ensino Fundamental com intuito de analisar como o desempenho motor de crianças com Transtorno do Déficit de Atenção e Hiperatividade (TDAH) pode ser influenciado pela ocorrência simultânea do Transtorno do Desenvolvimento da Coordenação (TDC). Participaram do estudo 283 crianças, com a anuência dos responsáveis, nos quais foi aplicado o Movement Battery Assessment for Children. Nessa etapa, os responsáveis e professores responderam o questionário SNAP-IV. Após essa etapa, foram selecionadas 27 crianças divididas em dois grupos, $14 \mathrm{com}$ TDAH e 13 com TDAH/TDC. Para avaliar a motricidade global e fina, equilíbrio, esquema corporal, organização espacial e temporal, em ambos os grupos, foi utilizada a Escala de Desenvolvimento Motor. Como resultado, há a sugestão de que existem dificuldades de motricidade fina e equilíbrio no grupo com TDAH/TDC. Os achados demonstram a possibilidade de relação TDAH/TDC e indicam que problemas são intrínsecos ao TDAH e como a ocorrência simultânea de TDC pode influenciar o desempenho motor de escolares.

Borgmann (2013) buscou em sua dissertação investigar e analisar o ensino das modalidades Goalball e Voleibol Sentado no ambiente escolar. Para isso, objetivou especificamente realizar as seguintes ações: a) investigar na literatura as iniciativas existentes sobre a presença do esporte paralímpico na escola; b) analisar o ensino do Goalball na escola; c) analisar o ensino do Voleibol Sentado na escola. Os objetivos b e c foram analisados a partir da percepção dos professores de Educação Física. Utilizou entrevista com oito professores que utilizam o Goalball e o Voleibol Sentado em suas aulas do Ensino Fundamental. $O$ resultado da pesquisa gerou três artigos, um sobre 0 esporte paralímpico na escola, outro sobre o ensino do Goalball na escola e o último sobre o ensino do Voleibol Sentado na escola. De modo geral, o estudo identificou grande possibilidade para a introdução de esportes paralímpicos em ambiente escolar, a partir de adaptações de estratégias de ensino e de materiais, visto a dificuldade de se conseguir alguns deles. A diversidade de oferta das atividades e modalidades esportivas torna as aulas de educação física escolar muito atraente para os alunos. Além de favorecer a desmistificação da deficiência como impeditivo da participação de todos. Esses resultados, apesar de os professores não terem experiências prévias com 
DOI: $10.12957 /$ e-mosaicos.2020.42690

as modalidades em questão, foram alcançados pela inovação das propostas de atividades, contando para isso com o fator criatividade dos docentes envolvidos.

\subsection{Categoria de estudos propositivos}

Os três estudos que se configuraram como categoria estudos propositivos foram dois em nível de mestrado e um de doutorado. Suas caracterizações e seus resultados são apresentados a seguir.

Cunha (2013), em sua dissertação de mestrado, objetivou descrever e analisar o processo de sistematização e transmissão do esporte adaptado como conteúdo de ensino nas aulas de Educação Física na escola, e, também, identificar e analisar em que medida a prática dos esportes adaptados nas aulas pode favorecer o processo de inclusão das pessoas com deficiência na escola. Para alcançar esses objetivos, o autor optou por um estudo propositivo em uma escola da rede pública de ensino, especificamente com alunos de ambos os sexos de 10 a 11 anos da $5^{a}$ série do Ensino Fundamental. Portanto, se caracteriza como um estudo de caso, no qual o autor propôs uma intervenção de 17 aulas, distribuídas em duas por semana na educação física regular. O conteúdo das aulas foi tematizado a partir do esporte adaptado transposto didaticamente para um modelo pedagógico progressista da Educação Física. Incluíram-se também duas horas semanais para avaliação e planejamentos das ações. Para a coleta de dados, foram utilizadas entrevistas semiestruturadas, diário de campo, vídeos e fotos. Os resultados apontaram que a adaptação pedagógica dos conteúdos e estratégias de ensino baseado em uma perspectiva progressista permitia a fácil inclusão dos atores no processo de ensino aprendizagem e ainda, que essas estratégias aproximam os alunos com e sem deficiência nas atividades escolares.

A dissertação de Almada (2017) realizou um estudo em quatro escolas do município de Campinas em São Paulo, que mantinham convênio com o programa de atividade motora adaptada (PROAMA). Essa pesquisa objetivou analisar a possibilidade de inclusão do Goalball para alunos do quinto ano. A amostra do estudo foi composta por turmas do $5^{\circ}$ ano do Ensino Fundamental das quatro escolas conveniadas. Foram realizadas três aulas de Goalball nas quatro escolas considerando elementos lúdicos, analíticos, pré-desportivos e de socialização da turma. A abordagem pedagógica privilegiou a participação ativa dos alunos em todo o processo de ensinoaprendizagem. Para a coleta de dados, foi utilizada uma entrevista semiestruturada para os professores de Educação Física e outra para os alunos participantes das intervenções com o Goalball. Como resultado da intervenção, foi percebido uma mudança de comportamento dos alunos no sentido de aceitação, compreensão e interesse pelos esportes paralímpicos e pelas pessoas com deficiência. Conceitos e atitudes frente a pessoas com deficiência também se mostraram modificados positivamente, além da demonstração do interesse em se aproximar das modalidades de forma mais intensa. 
DOI: $10.12957 /$ e-mosaicos.2020.42690

A tese de Pasetto (2017) objetivou investigar os efeitos das dicas visual e cinestésica fornecidas em conjunto e separadamente, durante a aprendizagem de habilidade motora específica por alunos do Ensino Fundamental. A amostra se constituiu de 99 alunos de ambos os sexos, sendo $62 \mathrm{com}$ surdez neurossensorial bilateral (de moderadamente severa a anacusia) e 37 ouvintes, com idades entorno de 10 anos. Utilizou-se para o estudo a tarefa de arremesso do dardo de salão. A amostra foi dividida em três grupos distintos. Cada grupo foi designado de acordo com a dica específica: dica visual (SV); dica cinestésica (SC) e dica visual e cinestésica (SVC). E foram compostos por surdos e ouvintes. As ações foram realizadas em três etapas: Pré-teste, Aquisição e Pós-teste. Os dados foram analisados quanto à aquisição da habilidade de lançar o dardo de forma descritiva dos acertos individuais em relação aos erros. Os resultados apontaram que o grupo que recebeu a dica visual obteve desempenho melhor que os grupos que receberam a dica cinestésica e a dica visual e cinestésica. Portanto, a dica visual é melhor para promover a aprendizagem motora dos alunos com deficiência auditiva do que a dica cinestésica ou a dica visual conjugada à cinestésica. Pode-se ainda afirmar que os efeitos da dica visual são específicos para a aprendizagem de alunos com deficiência auditiva e o nível de conhecimento da Linguagem Brasileira de Sinais não influencia a ocorrência ou não da aprendizagem motora.

\subsection{CATEgoria DE ESTUdOS Mistos}

A categoria de estudos mistos obteve a ocorrência de apenas um estudo em nível de mestrado no recorte temporal avaliado. Segue abaixo sua caracterização e resultados.

Cataldi (2013) apresenta em sua dissertação um estudo diagnóstico realizado na literatura identificando os principais apontamentos históricos da Política de Educação Inclusiva brasileira. Também realiza um diagnóstico do perfil de professores de diversas regiões do Brasil, responsáveis por diferentes componentes curriculares da Educação Básica e Educação Superior, mapeia e categoriza as práticas pedagógicas dos professores de Educação Física na dimensão da educação inclusiva e mapeia, avalia e categoriza a produção sobre o tema em 26 periódicos indexados no Qualis, publicados de 2000 a 2012. Ainda na dissertação, a autora apresenta uma proposta de curso no formato de Educação a Distância (EAD) para a capacitação de professores de Educação Física da Educação Básica na perspectiva da educação inclusiva e faz uma proposta pedagógica de intervenção em educação física escolar na dimensão da inclusão.

\section{CONSIDERAÇÕES FINAIS}

Os esportes adaptados e os paralímpicos ofertados em ambiente escolar ainda são muito escassos. Esse cenário pode ser influenciado pela escassez de estudos que 
DOI: $10.12957 /$ e-mosaicos.2020.42690

se debruçam sobre o tema e também pela falta de oferta de disciplinas curriculares que tratam do tema de maneira adequada durante a formação inicial dos professores de Educação Física. Entretanto, percebe-se uma alteração inicial desse status a partir dos dados aqui apresentados.

O presente estudo de revisão objetivou analisar a produção acadêmica dos programas de pós-graduação strito sensu em Educação Física no Brasil que tratam do tema esporte adaptado na escola. Os estudos analisados foram divididos em três categorias de análise, a saber: Estudos diagnósticos; Estudos propositivos; Estudos mistos. Apresentaram ainda subcategorias como, Pesquisa de campo, Análise documental e Revisão da literatura. Foi possível perceber a maior ocorrência de estudos diagnósticos e com características predominantes de pesquisas de campo. Esse fato pode indicar a existência de lacunas no conhecimento sobre as características das diferentes deficiências e também dos processos pedagógicos para o ensino desse conteúdo na escola. Essas lacunas podem ser fruto de oferta reduzida, ou ausência, de disciplinas na graduação em Educação Física, ou ainda, pela falta de interesse dos docentes atuantes na Educação Básica.

Também foi identificado no estudo que as pesquisas desenvolvidas em nível de mestrado e doutorado somente se avolumam nos últimos anos, especificamente a partir de 2013. Cabe ainda destacar que esses estudos se concentram regionalmente em universidades do Sul e Sudeste do Brasil, demonstrando pouca capilaridade de projetos e linhas de pesquisa no território nacional que abordam o tema do esporte adaptado.

Tendo em vista o cenário descrito pelo presente estudo ainda se desenvolvem poucas iniciativas de pesquisas sobre o esporte adaptado e/ou esporte paralímpico em ambiente escolar no Brasil. Dessa forma, há espaço profícuo para novos estudos que se concentrem em propostas pedagógicas para a inserção dessas modalidades, paraolímpicas ou adaptadas, na escola, no sentido de promover a inclusão, a conscientização e a diversidade de práticas corporais para os diferentes personagens da Educação Básica.

\section{Referências}

ALMADA, R. R. Uma proposta de ensino do goalball nas escolas: a visão dos professores e alunos. Dissertação (mestrado), Universidade Estadual de Campinas, Faculdade de Educação Física. 2017.

BARDIN, L. Análise de conteúdo. 5. ed. Lisboa: Edições 70, 2009. 
DOI: $10.12957 /$ e-mosaicos.2020.42690

BORGMANN, T. Ensino do esporte paralímpico na escola: estudo a partir da visão dos professores. 2013. 134 f. Dissertação (Mestrado), Universidade Estadual de Campinas, Faculdade de Educação Física. 2013.

BRACHT, V.; SOARES, F. R. A educação física nas práticas e nos discursos "inclusivos": um paradoxo chamado inclusão escolar. In: CONGRESSO BRASILEIRO DE CIÊNCIAS DO ESPORTE - CONBRACE, 14, 2005, Porto Alegre. Anais Eletrônicos do XIV Conbrace, Porto Alegre, 2005.

BRASIL. CAPES. Distribuição de Programas de Pós-graduação no Brasil por Estado 2016. Disponível em: https://geocapes.capes.gov.br/geocapes/.

BRASIL. CAPES. Cursos de pós-graduação em educação física avaliados e reconhecidos 2018. Disponível em:

https://sucupira.capes.gov.br/sucupira/public/consultas/coleta/programa/quantitativo s/quantitativoAreaConhecimento.jsf?areaAvaliacao=21.

CANCELLA, A. S. A oferta do esporte para o aluno com deficiência no Instituto Federal do Paraná-IFPR, campus Paranaguá: um movimento de reprodução ou resistência? 2017. 144 f. Dissertação (Mestrado), Universidade Federal do Paraná, Curitiba. 2017.

CATALDI, C. L. Os caminhos percorridos pela Educação Física Inclusiva: uma reflexão a partir do perfil e propostas pedagógicas dos professores de Educação Física. 2013. 165 f. Dissertação (Mestrado), Universidade Federal de Juiz de Fora. 2013.

CARVALHO, N. S. A. Surdo, língua e cultura: as práticas esportivas, recreativas e corporais na educação inclusiva. 2013. 150 f. Dissertação (Mestrado), Universidade Salgado de Oliveira, Programa de Pós-graduação em Ciências da Atividade Física. 2013.

CUNHA, L. M. O esporte adaptado como conteúdo nas aulas de educação física. 2013. 176 f. Dissertação (Mestrado), Universidade Federal do Espírito Santo, Programa de Pós-Graduação em Educação Física do Centro de Educação Física e Desportos. 2013.

GORGATTI, M. G. Educação Física Escolar e Inclusão: uma análise a partir do desenvolvimento motor e social de adolescentes com deficiência visual e das atitudes dos professores. 2005. Tese (Doutorado), Escola de Educação Física e Esporte, Universidade de São Paulo. 2005.

GOULARDINS, J. B. Desempenho motor de crianças com transtorno do déficit de atenção e hiperatividade e transtorno do desenvolvimento da coordenação. 2016. 77 f. Tese (Doutorado), Universidade de São Paulo, Escola de Educação Física e Esporte. 2016. 
DOI: $10.12957 /$ e-mosaicos.2020.42690

LOPES, M. C.; FABRIS, E. H. Inclusão e Educação. Belo Horizonte: Autêntica, 2013.

PASETTO, S. C. Efeito do tipo de dica na aprendizagem de habilidades motoras de indivíduos surdos. 2017. 142 f. Tese (Doutorado), Universidade de São Paulo, Escola de Educação Física e Esporte. 2017.

PATATAS, J. M.; BOSSCHER, V.; LEGG, D. Understanding parasport: an analysis of the differences between able-bodied and parasport from a sport policy perspective. International Journal of Sport Policy and Politics. v.10, n.2, p.235-254, 2018.

Disponível em:

https://www.tandfonline.com/doi/pdf/10.1080/19406940.2017.1359649

SANTOS, V. A. P. Transtorno do desenvolvimento da coordenação: um transtorno a ser contextualizado na casa e na creche. 2017. 242 f. Tese (Doutorado), Universidade Estadual de Londrina, Maringá. 2017.

SILVA, E. A. G. Projeto paralimpíadas escolares: intenção, evolução, articulações e contribuições ao paradesporto educacional brasileiro. 2017. 138 f. Dissertação (Mestrado), UNIVERSIDADE FEDERAL DO PARANÁ, Curitiba.

SILVA, R. H. R.; SANTIAGO, T. V.; BERTONI, S. Inclusão de alunos com deficiência nas aulas de educação física dos Colégios de Aplicação: a perspectiva de seus professores. Polyphonía, v. 22, n. 1, jan./jun. 2011.

THOMAS, R. J.; NELSON, J. K.; SILVERMAN, S. J. Métodos de pesquisa em atividade física. 6ed. Porto Alegre: Artmed, 2012.

Recebido em 13 de maio de 2019 Aceito em 22 de setembro de 2019 com o direito de primeira publicação cedido à e-Mosaicos.

Os artigos publicados são de acesso público, de uso gratuito, com atribuição de autoria obrigatória, para aplicações de finalidade educacional e não-comercial, de acordo com o modelo de licenciamento Creative Commons adotado pela revista. 